\title{
Announcement of New Editor-in-Chief for the Korean Journal of Radiology
}

\author{
Yeon Hyeon Choe, MD, PhD
}

${ }^{1}$ Department of Radiology, Samsung Medical Center, Sungkyunkwan University School of Medicine, Seoul, Korea; ${ }^{2}$ HVSI Imaging Center, Heart Vascular Stroke Institute, Samsung Medical Center, Sungkyunkwan University School of Medicine, Seoul, Korea

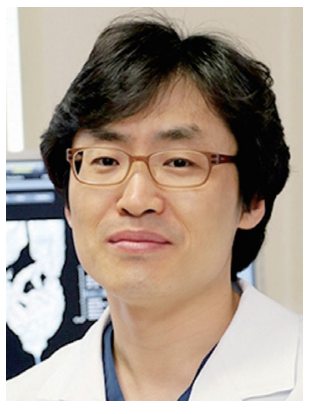

We are pleased to announce that Seong Ho Park, MD, PhD has been appointed as the new editor-in-chief for the Korean Journal of Radiology (KJR) by professor Joo Hyeong Oh, President of the Korean Society of Radiology (KSR), on behalf of KSR. His term begins in January 2021, replacing Yeon Hyeon Choe, MD, PhD, who is the current editor-in-chief.

Seong Ho Park, MD, PhD is a professor in the Department of Radiology and Research Institute of Radiology at Asan Medical Center, University of Ulsan College of Medicine, Korea. Dr. Park graduated from Seoul National University, College of Medicine in 1996. He received radiology residency training at Seoul National University Hospital and abdominal radiology fellowship training at Asan Medical Center.

Dr. Park is an experienced clinical practitioner and a world-renowned scholar in the field of gastrointestinal radiology. He has published numerous research papers in the academic area, particularly regarding the imaging-based diagnosis of colorectal cancer and inflammatory bowel diseases. Dr. Park also has extensive expertise in clinical research methodology and biostatistics. Most importantly, Dr. Park has vast experience as an editor or a peer reviewer for academic journals, which distinguishes him as an excellent candidate for the editor-in-chief for KJR among prolific scholars in the field of radiology. Dr. Park has served or has been serving as a deputy editor for KJR (2008-present), an associate editor for Radiology (2012-2017; Gastrointestinal Imaging section: 2019-present; Artificial Intelligence and Radiomics section), an assistant editor for the American Journal of Roentgenology (2014-2020), and a consultant to the editor for Radiology (2018). I believe that Dr. Park will be an excellent asset for KJR who will facilitate further improvements in the quality and influence of the journal. 